\title{
GLOBAL LOAD BALANCING ALGORITHM FOR WIRELESS MESH NETWORK
}

\author{
MOJTABA SEYEDZADEGAN \\ Faculty of electrical and computer engineering buein zahra technical university, buein zahra, qazvin, iran \\ E-mail: zadegan@bzte.ac.ir
}

\begin{abstract}
Unfortunately, current standards and protocols of Wireless Mesh Networks (WMNs) are severely inadequate to achieve the architectural design goals of wireless backbone mesh networks because they do not meet evolving network performance and cost requirements. Optimal placement of gateways provides acceptable network bandwidth for mesh users with reasonable investment costs. The focus in this paper is on the performance optimization for a formed Backbone WMN by improving the load balance between different gateway clusters (domains).
\end{abstract}

\section{INTRODUCTION}

WMNs have deployed many places in the last few years. The main reason for the development of WMN is to provide high bandwidth Internet access with high QoS and low costs for end users in any place at any moment. Before the advent of the IEEE 802.11 standards, wireless networks was for cellular long distance and communication with low data rate. The IEEE 802.11 protocol brought wireless networking to a new level through the use of unlicensed bands (2.4 $\mathrm{GHz}$ and $5.8 \mathrm{GHz}$ ) [1]. The physical layer specification (e.g. Multiple- Input Multiple-Output (MIMO) antennas) allowed for higher bandwidth than what was previously possible and being on the unlicensed band, regular consumers can use it. A WMN is a network of nodes connected by wireless communication links. It is a paradigm shift from the traditional centralized wireless systems such as cellular networks and Wireless Local Area Networks (WLANs). WMNs consist of Mesh Clients (MCs) and WMRs as shown in Fig 1. WMRs communicate in a multi-hop fashion forming the backbone of WMNs, where mesh routers have minimal mobility. In the most typical form of WMNs, every router performs relaying of data for other mesh routers. WMNs greatly help the users to be always-on-line all over no matter when by connecting to WMRs [2]. Moreover, certain mesh routers have the bridging functionality to connect WMNs to various existing wire and wireless networks such as cellular, wireless sensor, Wireless- Fidelity (Wi-Fi), Worldwide interoperability for Microwave Access (WiMAX), and Wireless multi-Media (WiMedia) networks. Thus, WMNs will deliver wireless services for a large variety of applications. WMN is a promising wireless technology for several applications [2], which include Metropolitan Area Networks (MAN), broadband home networking, enterprise networking, coordinated network management, community and neighborhood networks, intelligent transportation systems, health and medical systems, building automation, and security surveillance systems. It is gaining significant attention as a possible

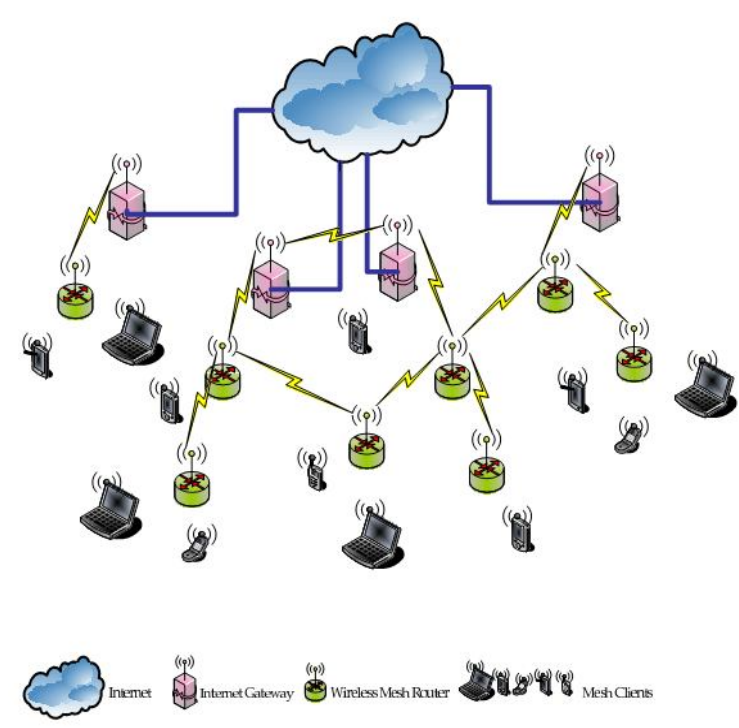

Fig. 1: Wireless Mesh Network

way for Internet Service Providers (ISPs) [3], [4] to provide robust and reliable wireless broadband service access with low investments. It also provides an alternative broadband solution for some scenarios where they are not easy to install the WLAN access points, or areas where the network connection is only required for a temporary period (e.g. disaster site). Although wireless mesh networks bring several advantages as compared to traditional wireless networks, there exist several open research issues that need to be investigated. There are challenges like: energy management, mobility management, capacity management, addressing and routing, integration with the Internet, and service levels, at different layers such as physical, Media Access Control (MAC), transport, and network. Many of these aspects have been discussed in [2] and [5]. Since the main purpose of WMN is to provide broadband Internet access and QoS for end users over a large coverage area at low costs [2], the design of the network architecture should be addressed carefully [6] because it is a fundamental issue for a WMN and is critical in determining the network performance and providing QoS for end users. 


\section{PROBLEM STATEMENTS}

The design of WMN includes some issues such as interface configuration,WMRs placement and gateways placement. WMR's throughput capacity and the Internet throughput, are considerably determined by the interface configuration. Since wireless radio costs are decreasing now, it is a feasible option to equip each WMR with multiple radio interfaces, so that the network capacity can be enhanced by assigning multiple orthogonal channels to each node. On the other hand, for WMRs and gateways placement, different locations of WMRs and gateways lead to different network topologies and architectures. For instance, moving an gateway to the left/right might removes it's right/left side WMRs from the gateway's transmission coverage. The removed WMRs, then, will join a new and nearest gateway. This leads to different network topology and architecture. Gateways are more expensive as compared to WMRs because they have to be specially configured to have access

to the Internet or to other networks. When gateways are not properly located, more gateways are needed to be placed in order to meet the full traffic requirements of WMRs. It causes the investment cost to increase consequently. Therefore, it is crucial to deploy the gateway at the right location so as to minimize the number of gateways while providing maximum capacity of WMN.

In a WMN, the traffic of a WMR is predominantly directed either towards the gateway or from the gateway to the WMR. Moreover, all the Internet traffic passes through gateways. Therefore, the MCs' throughput will be restricted by the gateways, even though the individual WMR can support a higher bandwidth. In addition to the gateway oriented Internet traffic, which taking into account by [7], unique gateways location factor, which is distributing gateways geographically close to wired network or Internet connection points, must be considered while designing the network architecture and deployment for a WMN.

\section{GATEWAY LOAD BALANCING}

As discussed before, a BWMN is divided into multiple gateway clusters in terms of the relationship between WMRs and gateway. Gateway is the head in each cluster. Since the traffic from the MCs in the WMN is directed between the WMRs and the wired network connection points or Internet, and all traffic passes through the gateways, gateways become the traffic centers in a BWMN. In the contention based media access protocols, such as: CSMA/CA in 802.11 [8], channel resource contention will be severe around gateway when traffic load is heavy. This will lead to the reduction of network performance, such as: higher packet loss probability and more packet delay.
Some gateways may only have low level of traffic from MCs while some others are heavily loaded. In order to reduce congestion around the heavily-loaded gateways and direct the WMRs traffic toward lightlyloaded gateways, a load balancing approach is needed. Under heavy traffic load situation, if some WMRs are able to change their connection (relationship) to another lightly-loaded gateway, channel utilization and average network performance will improve. Load balancing should be taken into account for increasing network throughput, avoiding congestion, and providing reliability [9], [10].

Load balancing in WMNs is an even more challenging problem due to the competition of wireless linksand limitations on available bandwidth. It can be classified into two categories: gateway and path load balancing. The path load balancing is for improving the network reliability and throughput by choosing paths with minimum channel interference, no traffic conflict, and lighter load [11]-[13].

Another important type of load balancing is the gateway load balancing. Gateway in a WMN, is a flows aggregation point and it could become a bottleneck due to the large number of radio interfaces and given channel bandwidth. Load balancing at gateways

have the greater performance improvement than at the path [7], [9]. In the gateway load balancing, the connection link between WMRs and gateways is changed based on the load of a particular gateway and the constraints of that particular cluster. To improve the load fairness between gateway clusters, a global load balancing approach is proposed to reassign the WMRs in a heavily-loaded gateway to a lighter-loaded gateway clusters. In other words, WMRs migrate from heavilyloaded gateway to lighter-loaded gateway clusters. In global load balancing approach, each gateway has information about neighbor's gateways and number of WMRs in each cluster in the network. Using this information, migration of WMRs from one cluster to another is possible subject to the constraints (i.e., delay, relay load, and cluster size) that are used to form such clusters. After the load balancing process, the WMN enters a state with a better fairness on traffic load.

\section{LOAD BALANCING APPROACH ILLUSTRATION}

An example of network topology, which consists of 24 nodes is considered and illustrated in Fig. 2a. There are three gateways (i.e., gw1, gw2, and gw3) in the sample network. It is initially partitioned into three service clusters,

$\mathrm{c} 1, \mathrm{c} 2$, and $\mathrm{c} 3$ with $\mathrm{c} 1=\{\mathrm{gw} 1, \mathrm{v} 1, \mathrm{v} 2, \mathrm{v} 3, \mathrm{v} 4, \mathrm{v} 5$, $\mathrm{v} 6\}, \mathrm{c} 2=\{\mathrm{gw} 2, \mathrm{v} 7, \mathrm{v} 8, \mathrm{v} 9, \mathrm{v} 10, \mathrm{v} 11, \mathrm{v} 12, \mathrm{v} 13, \mathrm{v} 14$, $\mathrm{v} 15\}$, and

$\mathrm{c} 3=\{\mathrm{gw} 3, \mathrm{v} 16, \mathrm{v} 17, \mathrm{v} 18, \mathrm{v} 19, \mathrm{v} 20, \mathrm{v} 21\}$, which is shown in Fig. 2b. With DQoS $=2$, WMRv7 is a bordering WMR 
among clusters $\mathrm{c} 1$ and $\mathrm{c} 2$, and WMRv15 is bordering WMR between clusters c2 and c3. two clusters are neighboring clusters if there exist some bordering nodes between them. By migrating the bordering WMR from one gateway cluster with heavier load to other lighter load cluster, the traffic load between different clusters can be re-distributed and get better load balancing. Fig. 2c shows three clusters after load balancing.

\section{GLOBAL LOAD BALANCING ALGORITHM}

The global load balancing algorithm (Algorithm 1) solves the gateway load balancing problem by iteratively identifying heavily-loaded gateways and re-assigning its' WMRs to lighter loaded identified gateways considering architectural design constraints. Optimum number of WMRs in each cluster is:

$$
O p t=\frac{n}{\sum_{g w \in G W} g w_{i}}
$$

where $\mathrm{n}$ is the total number of nodes in the network. In each iteration, an gateway (head of cluster) will be identified if its cluster size exceeds the Opt. WMRs from the identified gateway migrate to a neighbor cluster with lower cluster size than Opt subject to architectural design constraints. The iterative process ends when (i): there is no gateway with cluster size more than Opt or (ii): migration of WMRs from heavilyloaded gateway to lighter-loaded neigbor gateway is impossible due to architectural design constraints.
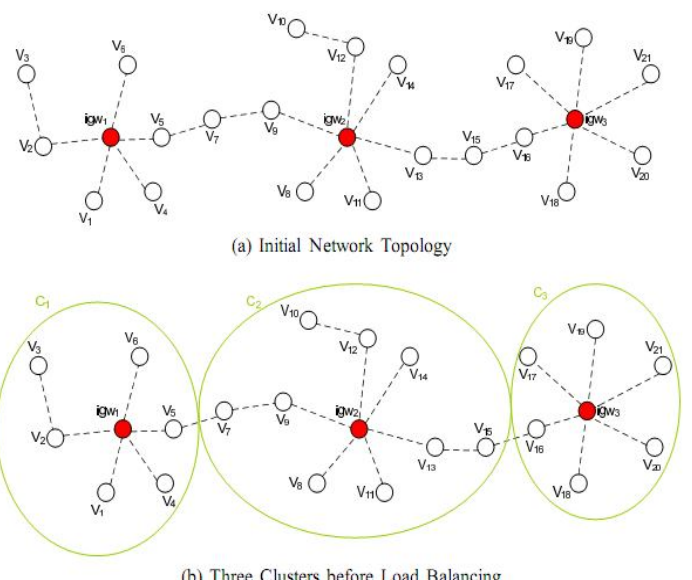

(b) Three Clusters before Load Balancing

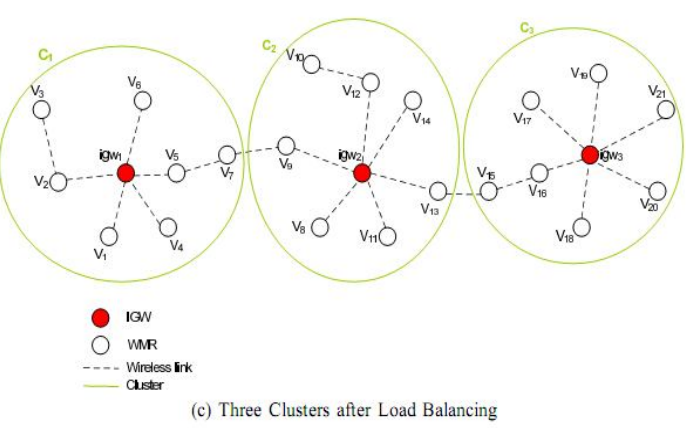

Fig. 2: Global Load Balancing Approach Illustration

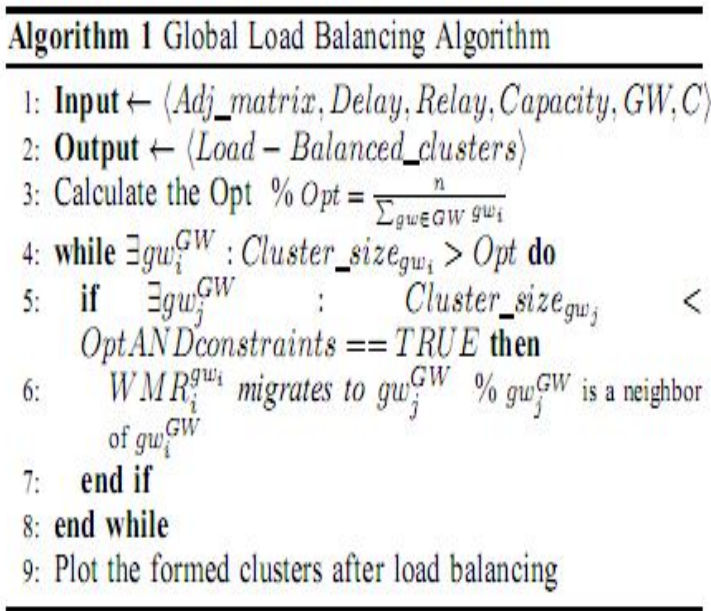

\section{LOAD BALANCING RESULTS DISCUSSION}

Load balancing is applied. Fig. 3 shows the improvement of load balancing using Jain's fairness index [14]. The number of nodes in each cluster is assumed as total load of that cluster. Therefore, optimum value of load per cluster is defined as total number of nodes in the network divided by total number of gateways (clusters). It is shown in Fig. 3 that the fairness index is close to 1 if the load balance algorithm is applied, which is interpreted to better and balanced traffic load in the backbone network. It is confirmed in conducted extensive simulation that delay, relay load, and capacity have no negative effect on load balancing approach. Thus, only effect of delay on load balancing is shown in Fig. 3

\section{CONCLUSION}

This paper elaborated on the importance of clustering for the efficient operation of BWMNs. The global load balancing approach provides better fairness in traffic loads among gateways.

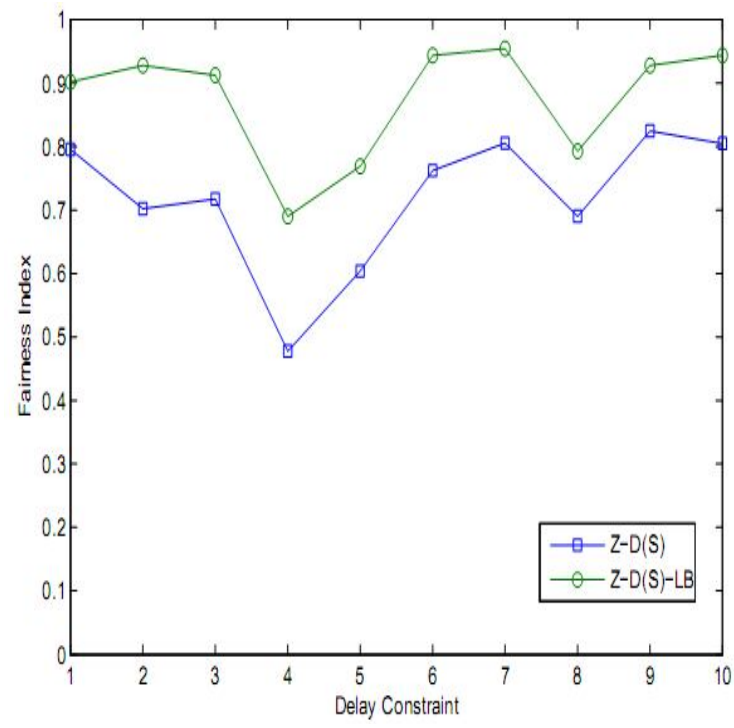

Fig. 3: Global Load Balancing approach 


\section{REFERENCES}

[1] IEEE Computer Society LAN MAN Standards Committee "Wireless LAN medium access control (MAC) and physical layer (PHY) specifications," 1997.

[2] I. Akyildiz, X. Wang, and W. Wang, "Wireless mesh networks: A survey," Computer Networks, vol. 47, no. 4, pp. 445-487, 2005.

[3] ArubaNetworks. (2011, access date: June 24, 2013) Nextgeneration wireless mesh networks: Combining a multiradio architecture with high performance routing to optimize video surveillance and other multimedia-grade applications. http://www.arubanetworks.com/pdf/technology/whitepapers/ wp wireless mesh. pdf. Aruba Networks. [Online]. Available: http://www.

arubanetworks.com/pdf/technology/whitepapers/wp wirelessmesh.pdf

[4] StrixSystems. (2013, access date: July 03, 2013) Wifi mesh business case. http://www.strixsystems.com/ casestudies/wifi-meshbusiness- case.asp. Strix Systems. [Online]. Available: http://www. strixsystems.com/case-studies/WiFiMesh-business-case.asp

[5] P. Pathak and R. Dutta, "A survey of network design problems and joint design approaches in wireless mesh networks," IEEE Communications Surveys and Tutorials, vol. 13, no. 3, pp. 396-428, 2011.

[6] B. He, "Architecture design and performance optimization of wireless mesh networks," Ph.D. dissertation, Computer Science and Engineering, College of Engineering, University of Cincinati, Ohio, 2010.
[7] B. He, B. Xie, and P. A. Dharma, "Optimizing deployment of internet gateway in wireless mesh networks," Computer Communications, vol. 31, no. 7, pp. 1259-1275, 2008.

[8] D. Agrawal and Q. Zeng, Introduction to Wireless and Mobile Systems. Thomson Engineering, 2010.

[9] K. Ramachandran, M. Buddhikot, G. Chandranmenon, S. Miller, E. Belding-Royer, and K. Almeroth, "On the design and implementation

of infrastructure mesh networks," in IEEE workshop on wireless mesh networks, ser. WiMesh '05, 2005, pp. 1-12.

[10] B. Xie, Y. Yu, A. Kumar, and D. P. Agrawal, "Loadbalanced mesh router migration for wireless mesh networks," Journal of Parallel and Distributed Computing, vol. 68, no. 6, pp. 825-839, 2008.

[11] V. Mhatre, H. Lundgren, F. Baccelli, and C. Diot, "Joint MAC-aware routing and load balancing in mesh networks," in 2007 ACM CoNEXT conference, ser. CoNEXT '07. ACM, 2007, p. 19.

[12] Y. Bejerano, S.-J. Han, and A. Kumar, "Efficient loadbalancing routing for wireless mesh networks," Computer Networks, vol. 51, no. 10, pp. 2450-2466, 2007.

[13] J. So and N. H. Vaidya, "Load-balancing routing in multichannel hybrid wireless networks with single network interface," IEEE Transactions on Vehicular Technology, vol. 56, no. 1, pp. 342-348, 2007.

[14] R. K. Jain, D.-M. W. Chiu, and W. R. Hawe, "A quantitative measure of fairness and discrimination for resource allocation in shared computer systems," DEC-TR-301, Digital Equipment Corporation, Tech. Rep., 1984 